\title{
Rhinology on the move despite Covid-19!
}

The October 2020 issue of Rhinology is a very interesting edition as it illustrates how world-wide colleagues pave the way for a better future of patients affected by nose and sinus diseases. After the successful launch of EPOS2020 in Spring 2020 (1), the editorial team of Rhinology is proud to present to you the latest and most exciting data in Rhinology research. Getting insight into the complexity and relevance of proteomics in CRS, epithelial-mesenchymal contribution to CRS, zinc levels in nasal and systemic compartment of CRS, nasal biomarkers of CRSwNP that predict recurrence of disease after sinus surgery, and the odor identification test for children, called 'U-Sniff', and FID scores (Frequency, Intensity and Duration) scores for epistaxis are all in the 2020 October issue and highly relevant for Rhinology practice. These studies build further on the solid grounds of previous Rhinology research meeting the unmets needs in the field ${ }^{(2-5)}$.

Spring 2020 is the era all of us will remember as the COVID-19 pandemic outbreak with smell dysfunction being more appreciated by society than before. Smell impairment has been underestimated as a major burden in CRS and other rhinology patient groups, and finally gets more attention as a significant symptom thanks to SARS-CoV-2 infected individuals who suffer from sudden onset smell loss and realize the burden. Olfactory adaptation and training in patients will olfactory deficits are dealt with by the group of Hummel ${ }^{(6)}$ and Klimek et al. ${ }^{(7)}$ suggest the inclusion of different kinds of novel digital tools into daily Rhinology practice, like artificial intelligence, telemedicine and distant patient monitoring.
The neural aspects of nasal breathing and patency have been neglected by research teams world-wide and finally receive more attention. Interestingly, the pre-operative evaluation of trigeminal sensitivity could be applied for improving patients' selection for septoplasty. Novel endoscopic surgical techniques and approaches allow better removal of skull base lesions, as highlighted in this issue. Authors also reconfirm the efficacy of nasal saline douching for persistent rhinitis as included in the guidelines ${ }^{(8)}$, the lack of correlation between PNIF values and OSAS severity, and other important aspects of our daily practice.

The Rhinology editorial team hopes you will get inspired by reading the October 2020 issue of Rhinology, and get motivated to do better for your patients.

\section{References}

1. Fokkens W, Desrosiers M, Harvey R, et al. EPOS2020: development strategy and goals for the latest European Position Paper on Rhinosinusitis. Rhinology. 2019 Jun 1;57(3):162-168.

2. Hoggard M, Zoing M, Biswas K, Taylor MW Douglas RG. The sinonasal mycobiota in chronic rhinosinusitis and control patients. Rhinology. 2019 Jun 1;57(3):190-199.

3. Khan A, Vandeplas G, Huynh TMT, et al. The Global Allergy and Asthma European Network (GALEN rhinosinusitis cohort: a large European cross-sectional study of chronic rhinosinusitis patients with and without nasal polyps. Rhinology. 2019 Feb 1;57(1):32-42

4. Phillips KM, Talat R, Caradonna DS, Gray ST, Sedaghat AR. Quality of life impairment due to chronic rhinosinusitis in asthmatics is mediated by asthma control. Rhinology. 2019 Dec 1;57(6):430-435.

5. Zeng M, Wang $\mathrm{H}$, Liao $\mathrm{B}$, et al. Comparison of efficacy of fluticasone propionate versus clarithromycin for postoperative treatment of different phenotypic chronic rhinosinusitis: a randomized controlled trial Rhinology 2019 Apr 1;57(2):101-109.

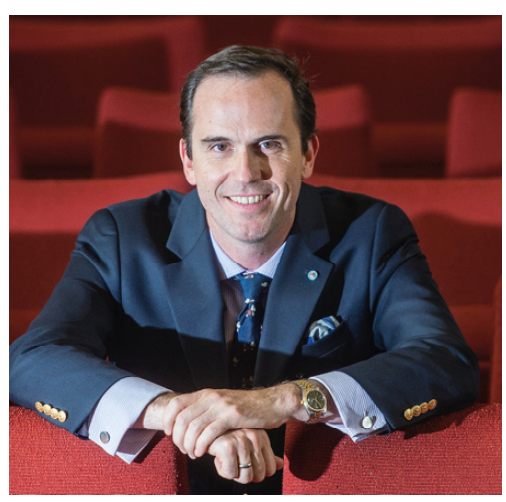

6. Chen B, Haehner A, Mahmut MK, Hummel T. Faster olfactory adaptation in patients with olfactory deficits: an analysis of results from odor threshold testing. Rhinology, 2020 Oct 1; 58(5):489-494.

7. Klimek L, Hagemann J, Alali A, et al. The toll of noninfected CRS patients to the Covid19 pandemic. Rhinology, 2020 Oct 1; 58(5): 522-523.

8. Hellings P, Scadding G, Bachert $C$, et al., EUFOREA treatment algorithm for allergic rhinitis. Rhinology 2020, in press. 